\title{
Dynamics and control of a free-floating space robot in presence of nonzero linear and angular momenta
}

\author{
Alessandro M. Giordano ${ }^{1,2}$, Gianluca Garofalo ${ }^{2}$, Marco De Stefano ${ }^{2}$, Christian Ott $^{2}$, Alin Albu-Schäffer ${ }^{1,2}$
}

\begin{abstract}
Common control methods for free-floating robots assume zero initial linear and angular momenta, for which a reduced joint dynamics equivalent to that of a fixed-base robot can be obtained. On the other hand, a disturbance is induced in the system dynamics when the linear or angular momenta are not zero, leading to a deviation of the end effector.

In this work the dynamics of the free-floating robot in presence of momentum is analyzed and a torque feedback control is proposed. An operational space formulation is considered to identify the disturbing Coriolis/centrifugal forces and to cancel them by feedback. A stability proof for the proposed controller is developed using a time-varying approach.

The effectiveness of the control is shown in simulation for a seven degrees-of-freedom arm connected to a floating-base under the effect of linear and angular momenta considering model parameters uncertainties.
\end{abstract}

\section{INTRODUCTION}

Space robots for on-orbit servicing missions involve the use of a robotic arm connected to a satellite for tasks such as refueling or de-orbiting of a malfunctioning target satellite. The aim of a so-called free-floating robot is to perform the tasks avoiding the use of the satellite actuators. In this way, nonrenewable fuel can be saved, increasing the life of the mission. For a free-floating robot, the motion of the satellite is dynamically coupled with the manipulator motion.

One important consequence of such coupling is the presence of dynamic singularities ([1], [2]) that are path-dependent, unlike for the fixed-base case. The presence of dynamic singularities further complicates the task of the trajectory planning algorithms [3]. In [4] the kinematic problem for a free-floating robot was addressed and the generalized Jacobian was presented. This relates the end effector velocities to the joint velocities, taking into account the conservation of momentum. Based on this concept, a kinematics-based control was then presented in [5]. The dynamics problem was treated in [6], where a transposed Jacobian approach was used for the end effector regulation and the stability was proven. The null-space dynamics of a redundant freefloating robot was first studied in [7], where the redundancy was resolved at velocity level.

The majority of the works on free-floating space robots assume zero initial linear and angular momenta of the system. Under this assumption, a reduced joint dynamics can be obtained, which is totally analogous to the one of a fixed-base

\footnotetext{
${ }^{1}$ The authors are with the Technical University of Munich (TUM), Chair of Sensor Based Robotic Systems and Intelligent Assistance Systems, Garching, 85748, Germany.

${ }^{2}$ The authors are with the Institute of Robotics and Mechatronics, German Aerospace Center (DLR), 82234 Weßling, Germany.

Contact alessandro.giordano@dlr.de
}

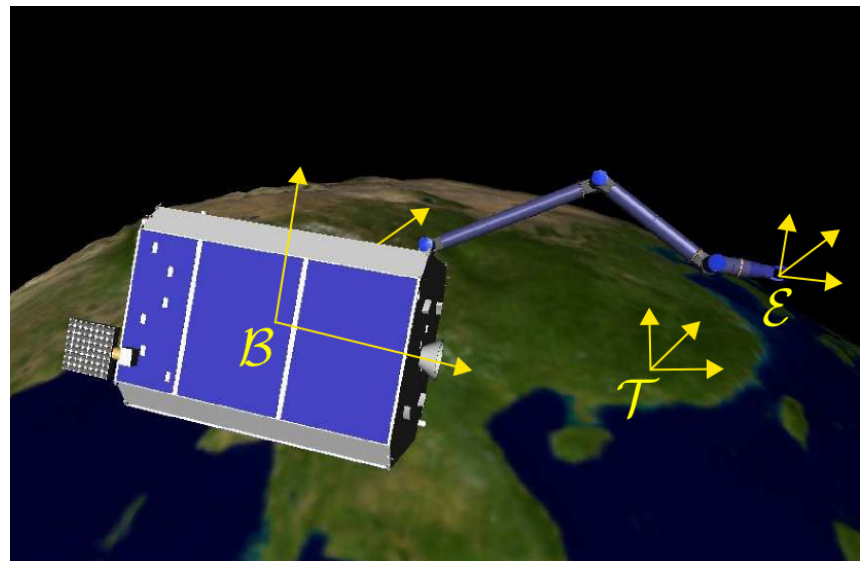

Fig. 1. Scenario and reference frames.

arm. On the other hand, when the initial momentum of the system is not zero, an additional dynamical term is induced in the equations in form of a persisting Coriolis/centrifugal force due to the momentum. During space operations, small amounts of momentum tend to accumulate, particularly for those on-orbit servicing scenarios where contacts are involved. Additionally, future missions might consider the use of an initial momentum to synchronize the motion of the servicer satellite with that of a tumbling target.

Up to now, a limited number of publications are available on this topic. The resolution of the redundancy at kinematic level considering initial momentum was analyzed in [7], whereas the tasks of base stabilization and end effector path tracking have been performed for the case of zero initial and angular momenta. In [8] a restricted workspace has been defined, where the end effector can remain fixed in space in presence of angular momentum. The trajectories of the system for some representative cases have been investigated. In [9] the same authors proposed a path planning approach considering initial angular momentum. In [10] an adaptive version of the Reaction Null-Space control was proposed at the kinematic level considering small linear and angular momenta. The work treated the linear and angular momenta as unknown quantities which are identified by an online parameter adaptation law. In [11] an impedance control for a free-floating robot was presented, based on a feedback linearization approach. In case of zero external force, the control in [11] reduces to the control of the free-floating robot in presence of momentum using feedback linearization. The exact feedback linearization provides the best theoretical performance. However, for practical implementation on 
torque controlled robots, robustness problems in presence of uncertain parameters and unmodeled dynamics could arise, limiting the performance [12].

In this paper, a torque control law for a free-floating robot in presence of momentum is proposed, that avoids the exact feedback linearization of the system. A suitable coordinate transformation is introduced in order to identify and remove the disturbance on the end effector caused by the momentum, leaving the inertia of the system unaltered. The proposed coordinated transformation further allows a stability analysis of the system. The effectiveness of the proposed control method has been tested in simulation for a seven degrees of freedom arm mounted on a mobile base considering dynamics parameters uncertainties.

In Section II the dynamics of a free-floating robot in presence of the momentum is analyzed for the case of nonredundant manipulator and the required compensation torque is derived in Section III In Section IV the results are extended to redundant manipulators. In Section $\mathrm{V}$ the stability proof for a redundant manipulator system is presented. Finally, the applicability of the controller is discussed in VI and the simulation results are shown in Section VII.

\section{FREE-FLOATING ROBOT IN PRESENCE OF MOMENTUM}

Let us consider a serial-link space robot composed of $n+1$ bodies, where $n$ is the number of joints of the robot. The system is floating in the inertial space with an initial momentum.

Let us consider the scenario where the robot end effector has to reach a target frame $\mathcal{T}$ attached on the orbit, as represented in Fig. 1. A base frame $\mathcal{B}$ is placed on the satellite CoM (Center of Mass) and a frame $\mathcal{E}$ is placed on the end effector of the robot.

For the small time scale of the robot maneuver, the orbital effects can be neglected and the frame $\mathcal{T}$ is considered inertial.

\section{A. Dynamics formulation}

In the absence of friction, the forward dynamics of the space robot is described by the equation [13]:

$$
\boldsymbol{H}(\boldsymbol{q}) \dot{\boldsymbol{v}}+\boldsymbol{S}(\boldsymbol{q}, \boldsymbol{v}) \boldsymbol{v}=\boldsymbol{f}
$$

The symbols used here are defined as follows:

$\boldsymbol{H} \in \mathbb{R}^{(6+n) \times(6+n)}$ inertia matrix of the free-floating robot, $\boldsymbol{S} \in \mathbb{R}^{(6+n) \times(6+n)}$ Coriolis and centrifugal matrix, $\boldsymbol{q} \in \mathbb{R}^{n}$ joint angles,

$\boldsymbol{v} \in \mathbb{R}^{6+n}$ generalized velocities $\left[\begin{array}{ll}\boldsymbol{\nu}_{b}^{T} & \dot{\boldsymbol{q}}^{T}\end{array}\right]^{T}$,

$\boldsymbol{\nu}_{b} \in \mathbb{R}^{6}$ base body twis $1^{1}\left[\begin{array}{ll}\boldsymbol{v}_{b}^{T} & \boldsymbol{\omega}_{b}^{T}\end{array}\right]^{T}$,

$\dot{\boldsymbol{q}} \in \mathbb{R}^{n}$ joint velocities,

$\boldsymbol{f} \in \mathbb{R}^{6+n}$ generalized forces $\left[\begin{array}{ll}\boldsymbol{f}_{b}^{T} & \boldsymbol{\tau}^{T}\end{array}\right]^{T}$,

$\boldsymbol{f}_{b} \in \mathbb{R}^{6}$ base wrench,

$\tau \in \mathbb{R}^{n}$ joint torques.

\footnotetext{
${ }^{1}$ The components $\boldsymbol{v}_{b} \in \mathbb{R}^{3}$ and $\boldsymbol{\omega}_{b} \in \mathbb{R}^{3}$ are respectively the body translational and angular velocity of the frame $\mathcal{B}$ w.r.t. the inertial frame. For the definition of the body twists and wrenches see [14].
}

Henceforth, the functional relation in the equations will be dropped out, except in the cases where it will be explicitly needed. Writing the symmetric inertia matrix in block diagonal form, it is

$$
\boldsymbol{H}=\left[\begin{array}{cc}
\boldsymbol{H}_{b} & \boldsymbol{H}_{b m} \\
\boldsymbol{H}_{b m}^{T} & \boldsymbol{H}_{m}
\end{array}\right],
$$

where $\boldsymbol{H}_{b} \in \mathbb{R}^{6 \times 6}$ is the locked inertia matrix [15] of the system, $\boldsymbol{H}_{b m} \in \mathbb{R}^{6 \times n}$ is the coupling inertia matrix and $\boldsymbol{H}_{m} \in \mathbb{R}^{n \times n}$ is the inertia matrix of the manipulator.

The end effector body twist $\nu_{e}$ is given by

$$
\boldsymbol{\nu}_{e}=\boldsymbol{J}(\boldsymbol{q}) \boldsymbol{v},
$$

where $\boldsymbol{J}=\left[\begin{array}{ll}\boldsymbol{J}_{b} & \boldsymbol{J}_{m}\end{array}\right] \in \mathbb{R}^{6 \times(6+n)}$ is the end effector Jacobian. The matrices $\boldsymbol{J}_{b} \in \mathbb{R}^{6 \times 6}$ and $\boldsymbol{J}_{m} \in \mathbb{R}^{6 \times n}$ are respectively the base and manipulator part of $\boldsymbol{J}$.

The momentum of the whole system, i.e. the sum of the momenta of each body, around the base reference frame $\mathcal{B}$, is given by [16]:

$$
\boldsymbol{h}_{b}=\boldsymbol{H}_{b} \boldsymbol{\nu}_{b}+\boldsymbol{H}_{b m} \dot{\boldsymbol{q}}
$$

being $\boldsymbol{h}_{b} \in \mathbb{R}^{6}$ composed of $\boldsymbol{h}_{b}=\left[\begin{array}{ll}\boldsymbol{h}_{b t}^{T} & \boldsymbol{h}_{b r}^{T}\end{array}\right]^{T}$, where $\boldsymbol{h}_{b t} \in \mathbb{R}^{3}$ and $\boldsymbol{h}_{b r} \in \mathbb{R}^{3}$ are respectively the linear and angular parts of the momentum. The momentum can be projected around the target frame $\mathcal{T}$ as

$$
\boldsymbol{h}=\boldsymbol{A}^{-T} \boldsymbol{J}_{b}^{-T} \boldsymbol{h}_{b}
$$

where $\boldsymbol{A}$ is the Adjoint matrix [17] from the inertial frame $\mathcal{T}$ to the end effector frame $\mathcal{E}$, defined as

$$
\boldsymbol{A}=\boldsymbol{A}(\tilde{\boldsymbol{x}})=\left[\begin{array}{cc}
\boldsymbol{R}_{t, e} & {\left[\boldsymbol{p}_{t, e}\right]^{\wedge} \boldsymbol{R}_{t, e}} \\
\mathbf{0}_{3 \times 3} & \boldsymbol{R}_{t, e}
\end{array}\right],
$$

where $\tilde{\boldsymbol{x}} \in \mathbb{R}^{6}$ is a local set of coordinates [14] for the position $\boldsymbol{p}_{t, e} \in \mathbb{R}^{3}$ and the rotation matrix $\boldsymbol{R}_{t, e} \in \mathbb{R}^{3 \times 3}$ of the end effector frame w.r.t. the inertial frame. The operator $[\cdot]^{\wedge}$ indicates the skew-symmetric matrix of the vector argument.

From (4) and (5) the momentum $\boldsymbol{h}$ can be written as a linear combination of the generalized velocities:

$$
\boldsymbol{h}=\boldsymbol{L}(\tilde{\boldsymbol{x}}, \boldsymbol{q}) \boldsymbol{v}=\boldsymbol{A}^{-T}(\tilde{\boldsymbol{x}}) \overline{\boldsymbol{L}}(\boldsymbol{q}) \boldsymbol{v}
$$

where $\overline{\boldsymbol{L}}=\boldsymbol{J}_{b}^{-T}\left[\begin{array}{ll}\boldsymbol{H}_{b} & \boldsymbol{H}_{b m}\end{array}\right]$. The coupled end effector/momentum dynamics is derived now using (7) and (3). A congruent coordinate transformation $\boldsymbol{T} \in \mathbb{R}^{12 \times(6+n)}$ [18] is introduced using the end effector twist $\nu_{e}$ and the momentum $\boldsymbol{h}$ :

$$
\left[\begin{array}{c}
\nu_{e} \\
h
\end{array}\right]=\left[\begin{array}{l}
J \\
L
\end{array}\right] v=T \boldsymbol{v} .
$$

In case of nonredundant manipulator and for nonsingular configurations it is possible to write the inverse transformation (see Appendix VIII-A):

$$
\boldsymbol{v}=\boldsymbol{T}^{-1}\left[\begin{array}{c}
\nu_{e} \\
\boldsymbol{h}
\end{array}\right]
$$


Applying the inverse of 8 on the dynamics in (1) and multiplying by $\boldsymbol{T}^{-T}$, is it possible to obtain the transformed dynamics matrices $\boldsymbol{M} \in \mathbb{R}^{12 \times 12}$ and $\boldsymbol{C} \in \mathbb{R}^{12 \times 12}$ as:

$$
\begin{aligned}
\boldsymbol{M} & =\boldsymbol{T}^{-T} \boldsymbol{H} \boldsymbol{T}^{-1}, \\
\boldsymbol{C} & =\boldsymbol{T}^{-T} \boldsymbol{S} \boldsymbol{T}^{-1}+\boldsymbol{T}^{-T} \boldsymbol{H} \frac{d}{d t} \boldsymbol{T}^{-1} .
\end{aligned}
$$

The generalized forces $f$ transform as

$$
\boldsymbol{f}=\boldsymbol{T}^{T}\left[\begin{array}{l}
\boldsymbol{f}_{e} \\
\boldsymbol{f}_{h}
\end{array}\right],
$$

where $\boldsymbol{f}_{e}, \boldsymbol{f}_{h} \in \mathbb{R}^{6}$ are the new control inputs. Notice that $\boldsymbol{f}_{e}, \boldsymbol{f}_{h}$ are dual respectively to $\boldsymbol{\nu}_{e}$ and $\boldsymbol{h}$. When the base is not actuated $\boldsymbol{f}_{b}=\mathbf{0}$ and (11) reduces to

$$
\boldsymbol{\tau}=\hat{\boldsymbol{J}}_{m}^{T} \boldsymbol{f}_{e},
$$

where $\hat{\boldsymbol{J}}_{m}=\boldsymbol{J}_{m}-\boldsymbol{J}_{b} \boldsymbol{H}_{b}^{-1} \boldsymbol{H}_{b m}$ is the well-known generalized Jacobian $\hat{\boldsymbol{J}}_{m}$ of the manipulator [4]. The transformed dynamics has the form:

$$
\begin{array}{r}
{\left[\begin{array}{cc}
\boldsymbol{M}_{e} & \boldsymbol{M}_{e h} \\
\boldsymbol{M}_{e h}^{T} & \boldsymbol{M}_{h}
\end{array}\right]\left[\begin{array}{c}
\dot{\boldsymbol{\nu}}_{e} \\
\dot{\boldsymbol{h}}
\end{array}\right]+\left[\begin{array}{cc}
\boldsymbol{C}_{e} & \boldsymbol{C}_{e h} \\
\boldsymbol{C}_{h e} & \boldsymbol{C}_{h}
\end{array}\right]\left[\begin{array}{c}
\boldsymbol{\nu}_{e} \\
\boldsymbol{h}
\end{array}\right]=} \\
{\left[\begin{array}{c}
\boldsymbol{f}_{e} \\
-\boldsymbol{A} \boldsymbol{J}_{b} \boldsymbol{H}_{b}^{-1} \boldsymbol{J}_{b}^{T} \boldsymbol{f}_{e}
\end{array}\right] .}
\end{array}
$$

In [19] the joint dynamics was shown to be decoupled from the momentum in the matrix $M$, while from (13) it is possible to see that this result does not hold for the end effector/momentum dynamics, that is fully coupled.

The force $\boldsymbol{f}_{e}$ is defined as an equivalent end effector force and it is actually an internal force. However, in (13) it appears also in the momentum equation. It can be proven that inserting the first equation of (13) into the second, the momentum equation results in $\dot{\boldsymbol{h}}=\mathbf{0}$. This is in agreement with the physical fact that the internal force $f_{e}$ can not cause an increase in the momentum of the total system.

\section{Momentum DeCOUPLING CONTROL}

The first row in (13) provides the end effector dynamics. Since the momentum is conserved $(\dot{\boldsymbol{h}}=\mathbf{0})$ the end effector equation simplifies to

$$
\boldsymbol{M}_{e}(\boldsymbol{q}) \dot{\boldsymbol{\nu}}_{e}+\boldsymbol{C}_{e}\left(\boldsymbol{q}, \boldsymbol{\nu}_{e}, \boldsymbol{h}\right) \boldsymbol{\nu}_{e}+\boldsymbol{C}_{e h}\left(\boldsymbol{q}, \tilde{\boldsymbol{x}}, \boldsymbol{\nu}_{e}, \boldsymbol{h}\right) \boldsymbol{h}=\boldsymbol{f}_{e},
$$

where, with an abuse of notation we indicated $\boldsymbol{C}_{e}\left(\boldsymbol{q}, \boldsymbol{\nu}_{e}, \boldsymbol{h}\right)$ with the same symbol after the change of the argument. The presence of an initial momentum $\boldsymbol{h} \neq \mathbf{0}$ induces a disturbance $\boldsymbol{C}_{e h} \boldsymbol{h}$ on the end effector dynamics (14), differently from the classical case of free-floating with zero initial momentum. When applying a simple PD control on the end effector, this term would produce a time varying ${ }^{2}$ error. The disturbance could be instead canceled by a control law

$$
\boldsymbol{f}_{e}=\boldsymbol{C}_{e h}\left(\boldsymbol{q}, \tilde{\boldsymbol{x}}, \boldsymbol{\nu}_{e}, \boldsymbol{h}\right) \boldsymbol{h}+\overline{\boldsymbol{f}}_{e},
$$

\footnotetext{
${ }^{2}$ This point will be further clarified later in Section VII
}

where $\bar{f}_{e}$ is the new control input. The decoupled dynamics can now be written as

$$
\boldsymbol{M}_{e}(\boldsymbol{q}) \dot{\boldsymbol{\nu}}_{e}+\boldsymbol{C}_{e}\left(\boldsymbol{q}, \boldsymbol{\nu}_{e}, \boldsymbol{h}\right) \boldsymbol{\nu}_{e}=\overline{\boldsymbol{f}}_{e} .
$$

Note that 16 does not depend on $\tilde{\boldsymbol{x}}$. A controller can be now designed for the end effector in order to reach a desired pose in the inertial frame as

$$
\overline{\boldsymbol{f}}_{e}=-\boldsymbol{J}_{\tilde{x} \nu}^{T}(\tilde{\boldsymbol{x}}) \boldsymbol{K} \tilde{\boldsymbol{x}}-\boldsymbol{D} \boldsymbol{\nu}_{e},
$$

where $\boldsymbol{K}, \boldsymbol{D} \in \mathbb{R}^{6 \times 6}$ are respectively symmetric positive definite matrices and $\boldsymbol{J}_{\tilde{x} \nu}(\tilde{\boldsymbol{x}}) \in \mathbb{R}^{6 \times 6}$ is the representation Jacobian [14] which correlates the error derivative $\dot{\tilde{\boldsymbol{x}}}$ and the end effector twist $\boldsymbol{\nu}_{e}$ :

$$
\dot{\tilde{\boldsymbol{x}}}=\boldsymbol{J}_{\tilde{x} \nu}(\tilde{\boldsymbol{x}}) \boldsymbol{\nu}_{e}
$$

From (17) and 16, the end effector equation is:

$$
\boldsymbol{M}_{e}(\boldsymbol{q}) \dot{\boldsymbol{\nu}}_{e}+\boldsymbol{C}_{e}\left(\boldsymbol{q}, \boldsymbol{\nu}_{e}, \boldsymbol{h}\right) \boldsymbol{\nu}_{e}+\boldsymbol{D} \boldsymbol{\nu}_{e}+\boldsymbol{J}_{\tilde{x} \nu}^{T}(\tilde{\boldsymbol{x}}) \boldsymbol{K} \tilde{\boldsymbol{x}}=\mathbf{0} .
$$

As it will be shown in Section $\mathrm{V}$, the end effector is asymptotically stable. In the following section, the approach is extended to the redundant manipulator case.

\section{REDUNDANT MANIPULATOR CASE}

In case of redundant manipulator with $r$ redundant degrees of freedom, the transformation matrix $T$ in $(8)$ can not be inverted. A solution is to augment the state [14] with a nullspace velocity $\boldsymbol{v}_{n}$, in order to get a square transformation matrix $\boldsymbol{T}_{N} \in \mathbb{R}^{(n+6) \times(n+6) \text { : }}$

$$
\left[\begin{array}{c}
\boldsymbol{\nu}_{e} \\
\boldsymbol{h} \\
\boldsymbol{v}_{n}
\end{array}\right]=\left[\begin{array}{c}
\boldsymbol{T} \\
\boldsymbol{N}
\end{array}\right] \boldsymbol{v}=\boldsymbol{T}_{N} \boldsymbol{v},
$$

where $\boldsymbol{N} \in \mathbb{R}^{r \times(6+n)}$ is a null-space matrix. The matrix $\boldsymbol{N}$ is defined using a null-space base matrix $\boldsymbol{Z} \in \mathbb{R}^{r \times(6+n)}$, i.e. satisfying

$$
\mathbf{T} \boldsymbol{Z}^{T}=\mathbf{0} .
$$

A consequence of the property (21) is that

$$
\left[\begin{array}{cc}
\boldsymbol{J}_{b} & \boldsymbol{J}_{m} \\
\boldsymbol{A}^{-T} \boldsymbol{J}_{b}^{-T} \boldsymbol{H}_{b} & \boldsymbol{A}^{-T} \boldsymbol{J}_{b}^{-T} \boldsymbol{H}_{b m}
\end{array}\right]\left[\begin{array}{c}
\boldsymbol{Z}_{b}^{T} \\
\boldsymbol{Z}_{m}^{T}
\end{array}\right]=\mathbf{0} \Rightarrow \hat{\boldsymbol{J}}_{m} \boldsymbol{Z}_{m}^{T}=\mathbf{0} .
$$

where the matrices $\boldsymbol{Z}_{b} \in \mathbb{R}^{r \times 6}$ and $\boldsymbol{Z}_{m} \in \mathbb{R}^{r \times n}$ are respectively the base and manipulator submatrices obtained reordering $\boldsymbol{Z}=\left[\begin{array}{ll}\boldsymbol{Z}_{b} & \boldsymbol{Z}_{m}\end{array}\right]$.

In order to prevent direct coupling of the null-space acceleration in the end effector acceleration and in the momentum equation, the matrix $N$ must be dynamically consistent, i.e. it must have the property $\boldsymbol{T} \boldsymbol{H}^{-1} \boldsymbol{N}^{T}=\mathbf{0}$. One can choose the null-space matrix [21] as

$$
\boldsymbol{N}=\left(\boldsymbol{Z} \boldsymbol{H} \boldsymbol{Z}^{T}\right)^{-1} \boldsymbol{Z} \boldsymbol{H} .
$$

Applying the inverse of (20) on the dynamics in (1) and multiplying by $\boldsymbol{T}_{N}^{-T}$, the transformed dynamics matrices

\footnotetext{
${ }^{3}$ One possible choice for the matrix $\boldsymbol{Z}$, as proposed in [20], is: $\boldsymbol{Z}=$ $\left[-\boldsymbol{T}_{r}^{T} \boldsymbol{T}_{s}^{-T} \boldsymbol{I}\right]$, where $\boldsymbol{T}_{s} \in \mathbb{R}^{12 \times 12}$ and $\boldsymbol{T}_{r} \in \mathbb{R}^{12 \times r}$ are respectively the square and redundant submatrices obtained reordering $\boldsymbol{T}=\left[\begin{array}{ll}\boldsymbol{T}_{s} & \boldsymbol{T}_{r}\end{array}\right]$.
} 
$\boldsymbol{M}_{N} \in \mathbb{R}^{(12+n) \times(12+n)}$ and $\boldsymbol{C}_{N} \in \mathbb{R}^{(12+n) \times(12+n)}$ can be obtained as

$$
\begin{aligned}
\boldsymbol{M}_{N} & =\boldsymbol{T}_{N}^{-T} \boldsymbol{H} \boldsymbol{T}_{N}^{-1}, \\
\boldsymbol{C}_{N} & =\boldsymbol{T}_{N}^{-T} \boldsymbol{S} \boldsymbol{T}_{N}^{-1}+\boldsymbol{T}_{N}^{-T} \boldsymbol{H} \frac{d}{d t} \boldsymbol{T}_{N}^{-1} .
\end{aligned}
$$

The generalized forces $f$ transform as

$$
\boldsymbol{f}=\boldsymbol{T}^{T}\left[\begin{array}{l}
\boldsymbol{f}_{e} \\
\boldsymbol{f}_{h} \\
\boldsymbol{f}_{n}
\end{array}\right],
$$

where $\boldsymbol{f}_{n} \in \mathbb{R}^{r}$ is a null-space force force dual to $\boldsymbol{v}_{n}$. When the base is not actuated $\boldsymbol{f}_{b}=\mathbf{0}$ and (25) reduces to

$$
\boldsymbol{\tau}=\hat{\boldsymbol{J}}_{m}^{T} \boldsymbol{f}_{e}+\hat{\boldsymbol{N}}^{T} \boldsymbol{f}_{n}
$$

where $\hat{\mathbf{N}}$ is the generalized null-space matrix of the freefloating, defined as

$$
\hat{\boldsymbol{N}}=\left(\boldsymbol{Z}_{m} \hat{\boldsymbol{H}}_{m} \boldsymbol{Z}_{m}^{T}\right)^{-1} \boldsymbol{Z}_{m} \hat{\boldsymbol{H}}_{m}
$$

where $\hat{\boldsymbol{H}}_{m}=\boldsymbol{H}_{m}-\boldsymbol{H}_{b m}^{T} \boldsymbol{H}_{b}^{-1} \boldsymbol{H}_{b m}$ is commonly indicated as "generalized inertia matrix" of the free-floating arm [22]. Notice that, similarly to the case of the transposed generalized Jacobian for the end effector force, a transposed generalized null-space matrix should be used for the null-space force of a free-floating robot. Considering the properties (21) and 22, the transformed dynamics can be written in the form

$$
\begin{gathered}
{\left[\begin{array}{ccc}
\boldsymbol{M}_{e} & \boldsymbol{M}_{e h} & \mathbf{0} \\
\boldsymbol{M}_{e h}^{T} & \boldsymbol{M}_{h} & \mathbf{0} \\
\mathbf{0} & \mathbf{0} & \boldsymbol{M}_{n}
\end{array}\right]\left[\begin{array}{c}
\dot{\boldsymbol{\nu}}_{e} \\
\dot{\boldsymbol{h}} \\
\dot{\boldsymbol{v}}_{n}
\end{array}\right]+\left[\begin{array}{ccc}
\boldsymbol{C}_{e} & \boldsymbol{C}_{e h} & \boldsymbol{C}_{e n} \\
\boldsymbol{C}_{h e} & \boldsymbol{C}_{h} & \boldsymbol{C}_{h n} \\
\boldsymbol{C}_{n e} & \boldsymbol{C}_{n h} & \boldsymbol{C}_{n}
\end{array}\right]\left[\begin{array}{c}
\boldsymbol{\nu}_{e} \\
\boldsymbol{h} \\
\boldsymbol{v}_{n}
\end{array}\right]} \\
=\left[\begin{array}{c}
\boldsymbol{f}_{e} \\
-\boldsymbol{A} \boldsymbol{J}_{b} \boldsymbol{H}_{b}^{-1} \boldsymbol{J}_{b}^{T} \boldsymbol{f}_{e} \\
\boldsymbol{f}_{n}
\end{array}\right] .
\end{gathered}
$$

From 28) it is possible to see that the null-space velocity $\boldsymbol{v}_{n}$ is inertially decoupled from the rest of the state, as a consequence of the dynamically consistent null-space matrix chosen in 23). However, the system is still fully coupled in the Coriolis/centrifugal matrix $\boldsymbol{C}_{N}$. In particular, it is again possible to note a coupling of the momentum $\boldsymbol{h}$ both on the end effector and the null-space dynamics by means of the terms $\boldsymbol{C}_{e h}$ and $\boldsymbol{C}_{n h}$. Furthermore, in the redundant case, an additional term $\boldsymbol{C}_{e n}$ couples the null-space in the end effector. Similarly to the nonredundant case, inserting the first equation of 28 into the second one, $\boldsymbol{h}=\mathbf{0}$ is obtained. A momentum decoupling law can now be defined such that

$$
\begin{aligned}
& \boldsymbol{f}_{e}=\boldsymbol{C}_{e h} \boldsymbol{h}+\boldsymbol{C}_{e n} \boldsymbol{v}_{n}+\overline{\boldsymbol{f}}_{e}, \\
& \boldsymbol{f}_{n}=\boldsymbol{C}_{n h} \boldsymbol{h}+\boldsymbol{C}_{n e} \boldsymbol{\nu}_{e}+\overline{\boldsymbol{f}}_{n},
\end{aligned}
$$

where $\bar{f}$ and $\bar{f}_{n}$ are the new control inputs. The control law 29) cancels the effect of the momentum $\boldsymbol{h}$ in the system and further decouples the reciprocal effect between the end effector and the null-space motion. A PD control law can then be chosen in order to regulate a desired end effector position, while damping out the null-space motion $\boldsymbol{v}_{n}$ :

$$
\begin{aligned}
& \overline{\boldsymbol{f}}_{e}=-\boldsymbol{J}_{\tilde{x} \nu}^{T} \boldsymbol{K} \tilde{\boldsymbol{x}}-\boldsymbol{D} \boldsymbol{\nu}_{e} \\
& \overline{\boldsymbol{f}}_{n}=-\boldsymbol{D}_{n} \boldsymbol{v}_{n}
\end{aligned}
$$

The end effector and null-space equations obtained from (28), (29) and (30) therefore are

$$
\begin{array}{r}
\boldsymbol{M}_{e}(\boldsymbol{q}) \dot{\boldsymbol{\nu}}_{e}+\boldsymbol{C}_{e}(\boldsymbol{q}, \boldsymbol{\chi}) \boldsymbol{\nu}_{e}+\boldsymbol{D} \boldsymbol{\nu}_{e}+\boldsymbol{J}_{\tilde{x} \nu}^{T}(\tilde{\boldsymbol{x}}) \boldsymbol{K} \tilde{\boldsymbol{x}}=\mathbf{0} \\
\boldsymbol{M}_{n}(\boldsymbol{q}) \dot{\boldsymbol{v}}_{n}+\boldsymbol{C}_{n}(\boldsymbol{q}, \boldsymbol{\chi}) \boldsymbol{v}_{n}+\boldsymbol{D}_{n} \boldsymbol{v}_{n}=\mathbf{0}
\end{array}
$$

where $\chi^{T}=\left[\boldsymbol{\nu}_{e}^{T}, \boldsymbol{h}^{T}, \boldsymbol{v}_{n}^{T}\right] \in \mathbb{R}^{12+r}$ for brevity.

In the following section, the stability proof will be addressed.

\section{STABILITY ANALYSiS}

A stability analysis for the redundant system is developed in the following, where the nonredundant case can be obtained as a subcase removing $\boldsymbol{v}_{n}$ from the proof.

Let us define a region $Q$ which excludes the singularities of the generalized Jacobian $\hat{\boldsymbol{J}}_{m}$ :

$$
Q=\left\{\boldsymbol{q} \in \mathbb{R}^{n}: \sigma_{\min }\left(\hat{\boldsymbol{J}}_{m}(\boldsymbol{q})\right)>\sigma_{0}>0\right\},
$$

where $\sigma_{\text {min }}=\inf _{\boldsymbol{q} \in \Omega} \hat{\boldsymbol{J}}_{m}(\boldsymbol{q})$ is the minimum possible singular value of $\hat{\boldsymbol{J}}_{m}(\boldsymbol{q})$. In the set $Q$ the inverse $\boldsymbol{T}_{N}^{-1}$ of the transformation 20 is bounded and the standard properties of the dynamics matrices $\boldsymbol{H}(\boldsymbol{q})$ and $\boldsymbol{S}(\boldsymbol{q}, \boldsymbol{v})$ can be extended [14] to the transformed matrices $\boldsymbol{M}_{N}(\boldsymbol{q}), \boldsymbol{C}_{N}(\boldsymbol{q}, \boldsymbol{\chi})$.

Considering a robot model with revolute joints, the following properties are considered [13],[23]:

Property 1. The matrix $\boldsymbol{M}_{N}(\boldsymbol{q})$ is symmetric and positive definite for all $\boldsymbol{q} \in Q$.

Property 2. The matrix $\boldsymbol{C}_{N}(\boldsymbol{q}, \boldsymbol{\chi})$ and the time derivative $\dot{M}_{N}(q)$ for all $\boldsymbol{q} \in Q, \chi \in \mathbb{R}^{12+r}$ satisfy

$$
\chi^{T}\left[\dot{\boldsymbol{M}}_{N}(\boldsymbol{q})-2 \boldsymbol{C}_{N}(\boldsymbol{q}, \boldsymbol{\chi})\right] \chi=\mathbf{0} .
$$

Property 3. There exist positive constants $\lambda_{M n, m i n}$, $\lambda_{M n, \max }, \gamma_{C n}, \gamma_{C n d 1}, \gamma_{C n d 2}$ such that for all $\boldsymbol{q} \in Q$, $\chi \in \mathbb{R}^{12+r}$ it is

$$
\begin{array}{r}
\lambda_{M n, \min } \leq\left\|\boldsymbol{M}_{N}(\boldsymbol{q})\right\| \leq \lambda_{M n, \max } \\
\left\|\boldsymbol{C}_{N}(\boldsymbol{q}, \boldsymbol{\chi})\right\| \leq \gamma_{C_{N}}\|\boldsymbol{\chi}\| \\
\left\|\dot{\boldsymbol{C}}_{N}(\boldsymbol{q}, \boldsymbol{\chi})\right\| \leq \gamma_{C_{N} d 1}\|\boldsymbol{\chi}\|+\gamma_{C_{N} d 2}\|\dot{\boldsymbol{\chi}}\|
\end{array}
$$

Remark 1. The properties 1, 2, 3 hold also for the submatrices $M_{e}, M_{n}, C_{e}, C_{n}$.

Without loss of generality, let us further assume a quaternion representation for the error $\tilde{\boldsymbol{x}}$, which leads $\forall \tilde{\boldsymbol{x}} \in \mathbb{R}^{6}$, $\nu_{e} \in \mathbb{R}^{6}$ to

$$
\left\|\boldsymbol{J}_{\tilde{x} \nu}(\tilde{\boldsymbol{x}})\right\| \leq \mu_{1}, \quad\left\|\dot{\boldsymbol{J}}_{\tilde{x} \nu}(\tilde{\boldsymbol{x}})\right\| \leq \mu_{2}\left\|\boldsymbol{\nu}_{e}\right\| .
$$


with $\mu_{1}, \mu_{2}>0$.

In order to analyze the asymptotic stability of the system [31, let us rewrite it as a time-varying system of the form:

$$
\begin{aligned}
& \boldsymbol{M}_{e}(t) \dot{\boldsymbol{\nu}}_{e}+\boldsymbol{C}_{e}(t) \boldsymbol{\nu}_{e}+\boldsymbol{D} \boldsymbol{\nu}_{e}+\boldsymbol{K} \boldsymbol{J}_{\tilde{x} \nu}^{T}(\tilde{\boldsymbol{x}}) \tilde{\boldsymbol{x}}=\mathbf{0}, \\
& \boldsymbol{M}_{n}(t) \dot{\boldsymbol{v}}_{n}+\boldsymbol{C}_{n}(t) \boldsymbol{v}_{n}+\boldsymbol{D}_{n} \boldsymbol{v}_{n}=\mathbf{0} .
\end{aligned}
$$

In this form the joints position $\boldsymbol{q}$ has been removed from the equations and the state is $\boldsymbol{z}^{T}=\left[\begin{array}{lll}\tilde{\boldsymbol{x}}^{T} & \boldsymbol{\nu}_{e}^{T} & \boldsymbol{v}_{n}^{T}\end{array}\right] \in \mathbb{R}^{12+r}$

Remark 2. For $\boldsymbol{q} \in \Omega$ the properties 1, 2, 3 hold also for the time-varying system 36 .

In the following, some preliminary properties of the states of the system (36) are proven in Lemma 11 The Lemma is reported here without proof, that can be found in Appendix VIII-B. Then, in Proposition 1 the stability proof is addressed.

Lemma 1. If $\boldsymbol{q} \in Q$, then there exists a set $\Omega$ such that the states $\tilde{\boldsymbol{x}}, \boldsymbol{\nu}_{e}, \boldsymbol{v}_{n}$, the accelerations $\dot{\boldsymbol{\nu}}_{e}, \dot{\boldsymbol{v}}_{n}$ and the matrices $\dot{\boldsymbol{M}}_{N}(t), \dot{\boldsymbol{C}}_{N}(t)$ are bounded.

For the proof of the asymptotic stability of the end effector dynamics some results regarding the stability properties of time-varying systems are used [24].

Proposition 1. If $\boldsymbol{q} \in Q$, then $\boldsymbol{z}=\mathbf{0}$ is an asymptotically stable equilibrium point for the system 36 .

Proof. A time-varying function $V(t, \boldsymbol{z})$ can be defined as

$$
V(t, \boldsymbol{z})=\frac{1}{2} \boldsymbol{\nu}_{e}^{T} \boldsymbol{M}_{e}(t) \boldsymbol{\nu}_{e}+\frac{1}{2} \boldsymbol{v}_{n}^{T} \boldsymbol{M}_{n}(t) \boldsymbol{v}_{n}+\frac{1}{2} \tilde{\boldsymbol{x}}^{T} \boldsymbol{K} \tilde{\boldsymbol{x}}
$$

Let us call $\lambda_{B, \min }$ and $\lambda_{B, \max }$ respectively the minimum and maximum eigenvalue of a matrix $\boldsymbol{B}$. For $\boldsymbol{z} \in \Omega$, the function $V(t, \boldsymbol{z})$ is positive definite and decrescent, since it is lower and upper bounded respectively by two positive definite functions $V_{\min }(\boldsymbol{z})$ and $V_{\max }(\boldsymbol{z})$ :

$$
\begin{aligned}
& V_{\text {min }}(\boldsymbol{z})=\frac{1}{2} \lambda_{M e, \min } \dot{x}_{e}^{2}+\frac{1}{2} \lambda_{M n, \min } v_{n}^{2}+\frac{1}{2} \lambda_{K, \min } \tilde{x}^{2} \\
& V_{\text {max }}(\boldsymbol{z})=\frac{1}{2} \lambda_{M e, \max } \dot{x}_{e}^{2}+\frac{1}{2} \lambda_{M n, \max } v_{n}^{2}+\frac{1}{2} \lambda_{K, \max } \tilde{x}^{2}
\end{aligned}
$$

The time derivative of $V$ is negative semidefinite. In fact:

$$
\begin{aligned}
& \dot{V}(t, \boldsymbol{z})=\boldsymbol{\nu}_{e}^{T}\left(-\boldsymbol{C}_{e} \boldsymbol{\nu}_{e}-\boldsymbol{D} \boldsymbol{\nu}_{e}-\boldsymbol{J}_{\tilde{x} \nu}^{T} \boldsymbol{K} \tilde{\boldsymbol{x}}\right)+\frac{1}{2} \boldsymbol{\nu}_{e}^{T} \dot{\boldsymbol{M}}_{e} \boldsymbol{\nu}_{e}+ \\
& +\boldsymbol{v}_{n}^{T}\left(-\boldsymbol{C}_{n} \boldsymbol{v}_{n}-\boldsymbol{D}_{n} \boldsymbol{v}_{n}\right)+\frac{1}{2} \boldsymbol{v}_{n}^{T} \dot{\boldsymbol{M}}_{n} \boldsymbol{v}_{n}+\boldsymbol{\nu}_{e}^{T} \boldsymbol{J}_{\tilde{x} \nu}^{T} \boldsymbol{K} \tilde{\boldsymbol{x}}= \\
& \quad=\frac{1}{2} \boldsymbol{\nu}_{e}^{T}\left(\dot{\boldsymbol{M}}_{e}-2 \boldsymbol{C}_{e}\right) \boldsymbol{\nu}_{e}+\frac{1}{2} \boldsymbol{v}_{n}^{T}\left(\dot{\boldsymbol{M}}_{n}-2 \boldsymbol{C}_{n}\right) \boldsymbol{v}_{n}+ \\
& \quad-\boldsymbol{\nu}_{e}^{T} \boldsymbol{D} \boldsymbol{\nu}_{e}-\boldsymbol{v}_{n}^{T} \boldsymbol{D}_{n} \boldsymbol{v}_{n}=-\boldsymbol{\nu}_{e}^{T} \boldsymbol{D} \boldsymbol{\nu}_{e}-\boldsymbol{v}_{n}^{T} \boldsymbol{D}_{n} \boldsymbol{v}_{n} \leq 0 .
\end{aligned}
$$

where Property 2 has been used. Therefore, the system is (uniformly) stable.

The convergence can be proven by means of a double application of the Barbalat's lemma (see Appendix VIII-B).
To do so, we need to analyze the boundedness of $\ddot{V}(t, \boldsymbol{z})$. Applying Lemma 11, it exists a set $\Omega$ where $\boldsymbol{\nu}_{e}, \dot{\boldsymbol{\nu}}_{e}, \boldsymbol{v}_{n}, \dot{\boldsymbol{v}}_{n}$ are bounded and, accordingly:

$$
\begin{aligned}
|\ddot{V}(t, \boldsymbol{z})|=\left|-2 \boldsymbol{\nu}_{e}^{T} \boldsymbol{D} \dot{\boldsymbol{\nu}}_{e}-2 \boldsymbol{v}_{n}^{T} \boldsymbol{D}_{n} \dot{\boldsymbol{v}}_{n}\right| \\
\leq 2 c_{1} c_{4} \lambda_{D, \text { max }}+2 c_{3} c_{5} \lambda_{\text {Dn,max }},
\end{aligned}
$$

where $c_{1}, c_{3}, c_{4}, c_{5}>0$ are respectively the bounds of $\boldsymbol{\nu}_{e}, \boldsymbol{v}_{n}, \dot{\boldsymbol{\nu}}_{e}, \dot{\boldsymbol{v}}_{n}$, as derived in Appendix VIII-B.

Applying Barbalat's Lemma on $V(t, \boldsymbol{z})$ it results $\dot{V}(t, \boldsymbol{z}) \rightarrow$ 0 and accordingly $\boldsymbol{\nu}_{e} \rightarrow \mathbf{0}$ and $\boldsymbol{v}_{n} \rightarrow \mathbf{0}$, i.e. the end effector and nullspace velocities converge to zero.

Let us now take in consideration the second order time derivative $\ddot{\boldsymbol{\nu}}_{e}$ of the end effector twist, which can be obtained differentiating 36a):

$$
\begin{aligned}
\dot{\boldsymbol{M}}_{e} \dot{\boldsymbol{\nu}}_{e}+\boldsymbol{M}_{e} \ddot{\boldsymbol{\nu}}_{e}+ & \dot{\boldsymbol{C}}_{e} \boldsymbol{\nu}_{e}+\boldsymbol{C}_{e} \dot{\boldsymbol{\nu}}_{e}+\boldsymbol{D} \dot{\boldsymbol{\nu}}_{e} \\
& +\boldsymbol{K} \boldsymbol{J}_{\tilde{x} \nu}^{T} \boldsymbol{J}_{\tilde{x} \nu} \boldsymbol{\nu}_{e}+\boldsymbol{K} \dot{\boldsymbol{J}}_{\tilde{x} \nu}^{T} \tilde{\boldsymbol{x}}=\mathbf{0},
\end{aligned}
$$

where (18) has been used. Considering (35) and Lemma 1. also $\ddot{\nu}_{e}$ can be proven to be bounded. In fact:

$$
\begin{aligned}
& \left\|\ddot{\boldsymbol{\nu}}_{e}\right\| \leq\left\|\boldsymbol{M}_{e}^{-1}\right\|\left(\left\|\dot{\boldsymbol{M}}_{e}+\boldsymbol{C}_{e}+\boldsymbol{D}\right\|\left\|\dot{\boldsymbol{\nu}}_{e}\right\|+\right. \\
& \left.\quad+\left\|\dot{\boldsymbol{C}}_{e}+\boldsymbol{K} \boldsymbol{J}_{\tilde{x} \nu}^{T} \boldsymbol{J}_{\tilde{x} \nu}\right\|\left\|\boldsymbol{\nu}_{e}\right\|+\left\|\boldsymbol{K} \dot{\boldsymbol{J}}_{\tilde{x} \nu}\right\|\|\tilde{\boldsymbol{x}}\|\right) \leq c_{6} .
\end{aligned}
$$

with $c_{6}>0$. Applying Barbalat's Lemma on $\boldsymbol{\nu}_{e}$, it follows that $\dot{\boldsymbol{\nu}}_{e} \rightarrow \mathbf{0}$.

The convergence of the end effector error $\tilde{\boldsymbol{x}}$ can be finally shown doing the limit of equation 36a:

$$
\lim _{t \rightarrow \infty}\left[\boldsymbol{M}_{e}(t) \dot{\boldsymbol{\nu}}_{e}+\boldsymbol{C}_{e}(t) \boldsymbol{\nu}_{e}+\boldsymbol{D} \boldsymbol{\nu}_{e}+\boldsymbol{K} \boldsymbol{J}_{\tilde{x} \nu}^{T}(\tilde{\boldsymbol{x}}) \tilde{\boldsymbol{x}}\right]=\mathbf{0}
$$

Considering (35), it follows $\tilde{\boldsymbol{x}} \rightarrow \mathbf{0}$. Then, the system (36) is asymptotically stable.

\section{CONTROLLER DISCUSSION}

Considering the inverse of 20 it is possible to see that, while the end effector and nullspace velocities go to zero, the coupled base-joints motion continues to evolve as a consequence of the constant generalized momentum $\boldsymbol{h}$ :

$$
\begin{aligned}
\boldsymbol{\nu}_{b} & \rightarrow \boldsymbol{J}_{b}^{-1} \boldsymbol{J}_{m} \hat{\boldsymbol{J}}_{m}^{\#} \boldsymbol{J}_{b} \boldsymbol{H}_{b}^{-1} \boldsymbol{J}_{b}^{T} \boldsymbol{A}^{T} \boldsymbol{h} \\
\dot{\boldsymbol{q}} & \rightarrow-\hat{\boldsymbol{J}}_{m}^{\#} \boldsymbol{J}_{b} \boldsymbol{H}_{b}^{-1} \boldsymbol{J}_{b}^{T} \boldsymbol{A}^{T} \boldsymbol{h}
\end{aligned}
$$

In case of zero initial momentum, the joint and base velocities converge to zero and the proposed control coincides with the classical transposed generalized Jacobian control [6].

\section{A. Limitation of the controller}

The convergence of the end effector is guaranteed only when the singularities of $\hat{\boldsymbol{J}}_{m}(\boldsymbol{q})$ are avoided. However, in presence of initial momentum, the singularities could be encountered also when the end effector has converged, due to the highlighted persistent joint motion. Considering for example the particular case of $\boldsymbol{h}_{t} \neq \mathbf{0}$, i.e. the system has a linear momentum, the joints will necessarily reach after a certain time a singular configuration. This result, observed 
also in [8], [11], is a natural consequence of the fact that it is not possible to control the end effector in the inertial frame for an indefinite time if the whole system is moving in space with a translational velocity. On the other hand, in the case of only angular momentum $\boldsymbol{h}_{t}=\mathbf{0}$, the problem of singularity could be less restrictive and singularity-free paths could be found. In this case, however, the robot will likely converge to joint limits, due to the continuous rotational joint motion induced by the angular momentum.

In conclusion, until a singularity is reached, the error will continuously decrease due to the proven convergence properties. In this way, the required end effector precision could be ensured during the time of the gripper closure. Additional measures need to be taken to avoid entering the singularities, for example by activating the satellite thrusters.

From a theoretical point of view, the controller has no assumption on the maximum amount of momentum that can be handled. However, in practice, for higher momenta, the smaller time to singularity will limit the applicability of the control and additional satellite control would be required

\section{B. Practical implementation}

The proposed controller is based on the cancellation of the disturbance term $\boldsymbol{C}_{e h} \boldsymbol{h}, \boldsymbol{C}_{e n} \boldsymbol{v}_{n}$ from the end effector dynamics. The matrices $\boldsymbol{C}_{e h}, \boldsymbol{C}_{e n}$ can be evaluated using an online dynamics implementation and the quantities $\boldsymbol{h}, \boldsymbol{v}_{n}$ can be calculated using (7), 20, as

$$
\begin{aligned}
\boldsymbol{h} & =\boldsymbol{L}_{b}(\tilde{\boldsymbol{x}}, \boldsymbol{q}) \boldsymbol{\nu}_{b}+\boldsymbol{L}_{m}(\tilde{\boldsymbol{x}}, \boldsymbol{q}) \dot{\boldsymbol{q}} \\
\boldsymbol{v}_{n} & =\boldsymbol{N}_{b}(\boldsymbol{q}) \boldsymbol{\nu}_{b}+\boldsymbol{N}_{m}(\boldsymbol{q}) \dot{\boldsymbol{q}}
\end{aligned}
$$

The base twist $\nu_{b}$ can estimated using (3):

$$
\boldsymbol{\nu}_{b}=\boldsymbol{J}_{b}^{-1}(\boldsymbol{q}) \boldsymbol{\nu}_{e}-\boldsymbol{J}_{b}^{-1}(\boldsymbol{q}) \boldsymbol{J}_{m}(\boldsymbol{q}) \dot{\boldsymbol{q}} .
$$

In this way the estimation of the momentum does not need any information about the satellite state but only joint $\boldsymbol{q}, \dot{\boldsymbol{q}}$ and end effector measurements $\tilde{\boldsymbol{x}}, \boldsymbol{\nu}_{e}$. The latter could be obtained using a tip mounted camera pointing a target object in the inertial frame.

\section{Simulation RESUlts}

A seven degrees-of-freedom free-floating robot in presence of linear and angular momenta is simulated, in order to verify the proposed control method. The kinematics and dynamics parameters of the robot are defined in Tab. I The initial joints configuration is $\boldsymbol{q}(0)=$ $[0,60,0,-60,0,30,0] \mathrm{deg}$, as schematically depicted in Fig. 1. The initial linear and angular momenta are respectively $\boldsymbol{h}_{b t}=[-0.8,-0.38,21.6] \mathrm{kg} \mathrm{m} / \mathrm{s}$ and $\boldsymbol{h}_{b r}=$ $[0.65,-19.63,0.87] \mathrm{kg} \mathrm{m}^{3} / \mathrm{s}$, corresponding to an initial linear velocity of the base $\boldsymbol{v}_{b}=[-2.0,1.0,53] \mathrm{mm} / \mathrm{s}$ and to an angular velocity of $\boldsymbol{\omega}_{b}=[-0.2,-3.3,0.2] \mathrm{deg} / \mathrm{s}$.

A step input $\Delta \boldsymbol{p}=[-3.5,0,-3] \mathrm{cm}, \Delta \boldsymbol{\phi}=[0,5,0] \mathrm{deg}$ on the end effector is commanded to the robot in the case of:

1) End effector PD control using the transposed generalized Jacobian [6], i.e.

$$
\boldsymbol{\tau}=\hat{\boldsymbol{J}}_{m}^{T}\left(-\boldsymbol{J}_{\tilde{x} \nu}^{T} \boldsymbol{K} \tilde{\boldsymbol{x}}-\boldsymbol{D} \boldsymbol{\nu}_{e}\right)
$$

TABLE I

PARAMETERS OF THE FREE-FLOATING ROBOT

\begin{tabular}{cccccc}
\hline & $l[\mathrm{~m}]$ & $m[\mathrm{~kg}]$ & $I_{x}\left[\mathrm{kgm}^{2}\right]$ & $I_{y}\left[\mathrm{kgm}^{2}\right]$ & $I_{z}\left[\mathrm{kgm}^{2}\right]$ \\
\cline { 2 - 6 } Base & 1.17 & 375 & 165 & 280 & 250 \\
Link 1 & 0.17 & 5.1 & 0.03 & 0.03 & 0.03 \\
Link 2 & 1.3 & 18.8 & 1.65 & 0.64 & 1.65 \\
Link 3 & 0.17 & 8.9 & 0.15 & 0.03 & 0.14 \\
Link 4 & 1.3 & 12.0 & 0.25 & 0.03 & 0.25 \\
Link 5 & 0.17 & 11.7 & 0.26 & 0.03 & 0.26 \\
Link 6 & 0.1 & 5.5 & 0.02 & 0.02 & 0.03 \\
Link 7 & 0 & 4.7 & 0.02 & 0.01 & 0.01 \\
\hline
\end{tabular}

2) Proposed momentum compensation control (Eq 26, (29), (30).

The stiffness and damping values used are respectively $K_{t}=165 \mathrm{~N} / \mathrm{m}$ and $D_{t}=175 \mathrm{Ns} / \mathrm{m}$ for the translation, $K_{r}=20 \mathrm{~N} / \mathrm{rad}$ and $D_{r}=20 \mathrm{Ns} / \mathrm{rad}$ for the rotation.

The norm of end effector position error is shown in Fig. 2 for both controllers. The joint angles and base attitude are shown for the proposed control respectively in Fig. 3 and Fig. 4 ,

The results show that for the proposed control the end effector converges to the desired position. On the other hand, a deviation on the end effector is induced in case that the momentum compensation is not used.

In general, this deviation is time-varying and its magnitude depends on both the initial momentum and on the particular maneuver commanded to the robot, since $\boldsymbol{C}_{e h}=\boldsymbol{C}_{e h}(\boldsymbol{q}(t))$. In fact, from Fig. 3 and Fig. 4 it is possible to notice that the joints and base motion continues to evolve while the end effector remains fixed, as a consequence of the presence of momentum. This explains the time-variability of the disturbance.

Finally, the convergence of the nullspace velocity $\boldsymbol{v}_{n}$ is represented in Fig. 5, showing the effectiveness of the

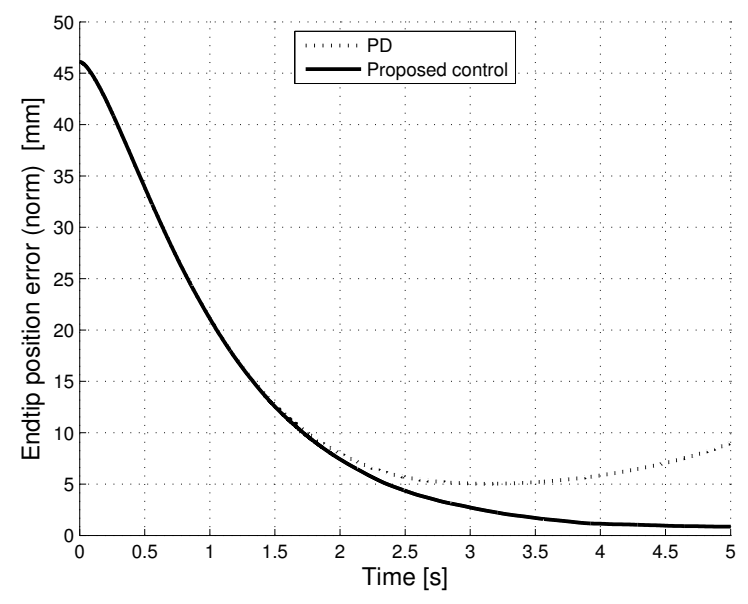

Fig. 2. Norm of the end effector position error for the PD control and for the proposed control. 


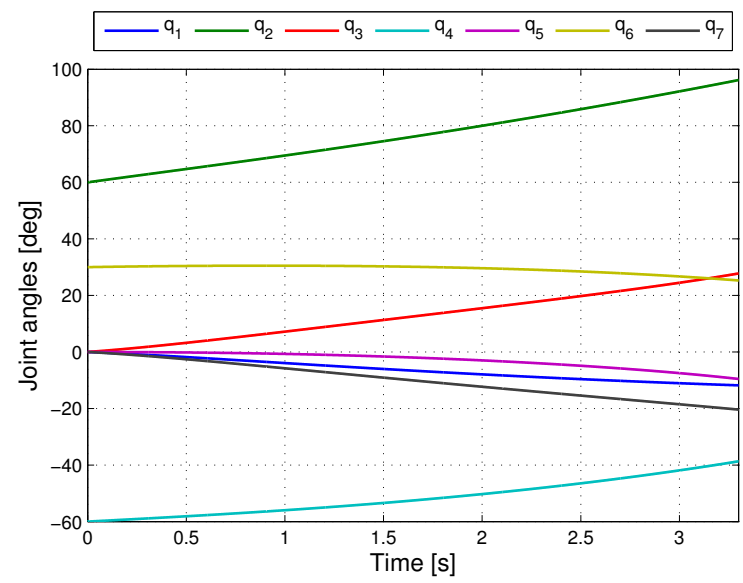

Fig. 3. Joint angles with the proposed control.

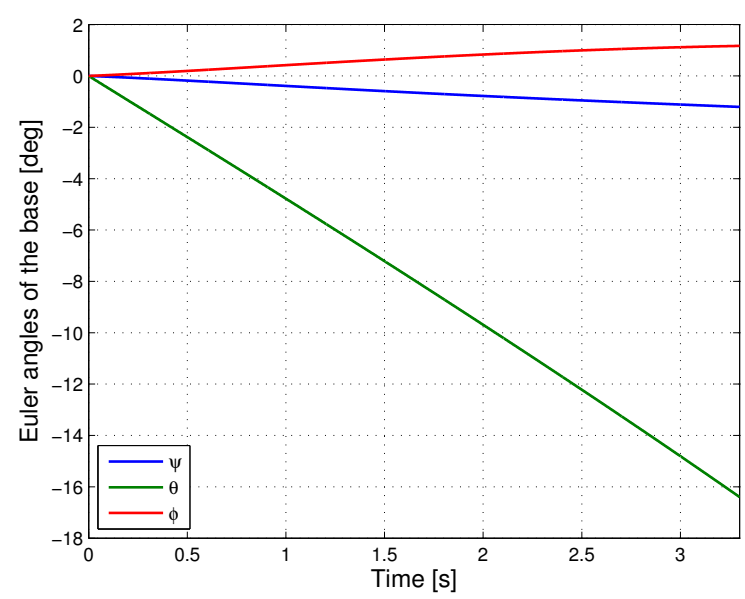

Fig. 4. Attitude of the base.

nullspace control.

Since the proposed control is based on the online dynamic model of the space robot, uncertainties in the dynamics parameters are introduced, in order to test the robustness. The proposed controller has been tested with 20 randomly generated models, considering for each body $10 \%$ uncertainty in the mass and $40 \%$ uncertainty in the inertias and in the CoMs. Fig. 6 shows an envelope of norm of the step response of the proposed controller with the uncertain models. The results show that the controller is robust considering dynamics parameters uncertainties and a worst-case deviation of 3.5 $\mathrm{mm}$ from the nominal behavior is obtained.

\section{CONCLUSIONS AND FUTURE WORKS}

In this work, the dynamics of a free-floating system in presence of linear and angular momenta has been investigated. It has been shown that the presence of the momentum introduces a drift on the end effector and a torque control law has been proposed in order to compensate that disturbance without exactly feedback linearizing the system. For

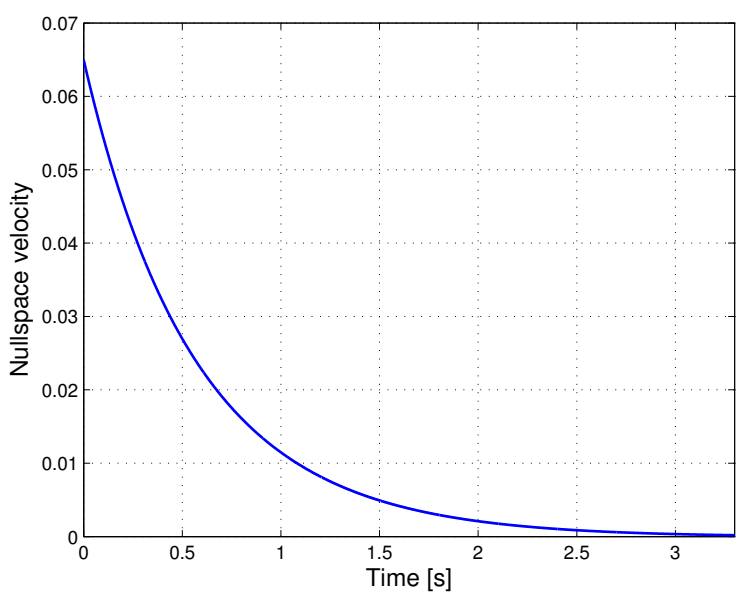

Fig. 5. Nullspace velocity with the proposed control.

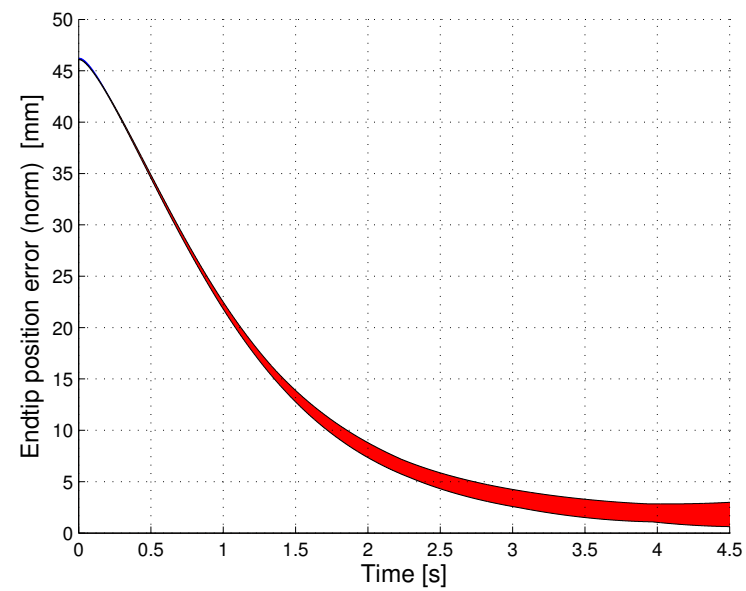

Fig. 6. Envelope of step response (norm) of the end effector for the proposed controller for 20 uncertain models.

the proposed controller a stability proof was given under the hypothesis of non-singularity of the space robot. The effectiveness of the control has been tested in simulation considering model uncertainties. Further investigations could focus on the study of the trajectories of a free-floating system under the effect of momentum in order to clarify whether is possible to find singularity-free paths where the controller behavior is well defined.

\section{APPENDIX}

\section{A. Inversion of the transformation $\mathbf{T}_{N}$}

The inverse transformation can be obtained [14] as $\boldsymbol{T}_{N}^{-1}=$ $\left[\begin{array}{ll}\boldsymbol{T}^{\#} & \boldsymbol{Z}^{T}\end{array}\right]$, calculating the weighted pseudoinverse $\boldsymbol{T}^{\#}=$ $\boldsymbol{H}^{-1} \boldsymbol{T}^{T}\left(\boldsymbol{T} \boldsymbol{H}^{-1} \boldsymbol{T}^{T}\right)^{-1}$ in explicit form:

$$
\boldsymbol{T}^{\#}=\left[\begin{array}{cc}
-\boldsymbol{H}_{b}^{-1} \boldsymbol{H}_{b m} \hat{\boldsymbol{J}}_{m}^{\#} & \boldsymbol{J}_{b}^{-1} \boldsymbol{J}_{m} \hat{\boldsymbol{J}}_{m}^{\#} \boldsymbol{J}_{b} \boldsymbol{H}_{b}^{-1} \boldsymbol{J}_{b}^{T} \boldsymbol{A}^{T} \\
\hat{\boldsymbol{J}}_{m}^{\#} & -\hat{\boldsymbol{J}}_{m}^{\#} \boldsymbol{J}_{b} \boldsymbol{H}_{b}^{-1} \boldsymbol{J}_{b}^{T} \boldsymbol{A}^{T}
\end{array}\right],
$$

being $\hat{\boldsymbol{J}}_{m}^{\#}=\hat{\boldsymbol{H}}_{m}^{-1} \hat{\boldsymbol{J}}_{m}^{T}\left(\hat{\boldsymbol{J}}_{m} \hat{\boldsymbol{H}}_{m}^{-1} \hat{\boldsymbol{J}}_{m}^{T}\right)^{-1}$ the weighted pseudoinverse of the generalized Jacobian $\hat{\boldsymbol{J}}_{m}$ with a weight matrix 
$\hat{\boldsymbol{H}}_{m}$. Notice that for nonredundant robot $\hat{\boldsymbol{J}}_{m}^{\#}=\hat{\boldsymbol{J}}_{m}^{-1}$ and $T^{\#}=T^{-1}$.

\section{B. Lemmas}

Lemma 1. If $\boldsymbol{q} \in Q$, then there exists a set $\Omega$ such that the states $\tilde{\boldsymbol{x}}, \boldsymbol{\nu}_{e}, \boldsymbol{v}_{n}$, the accelerations $\dot{\boldsymbol{\nu}}_{e}, \dot{\boldsymbol{v}}_{n}$ and the matrices $\dot{\boldsymbol{M}}_{N}(t), \dot{\boldsymbol{C}}_{N}(t)$ are bounded.

Proof. Let us consider the positive definite function $V(t, \boldsymbol{z})$ defined in (37). Choosing a constant $V_{0}>0$, let us a define a set $\Omega=\left\{\boldsymbol{z} \in \mathbb{R}^{12+r}: V(\boldsymbol{z}) \leq V_{0}\right\}$.

Considering that $\dot{V}(t, \boldsymbol{z}) \leq 0$, it follows that there exist $c_{1}, c_{2}, c_{3}>0$ such that $\forall \boldsymbol{z} \in \Omega$ it is

$$
\left\|\boldsymbol{\nu}_{e}\right\| \leq c_{1}, \quad\|\tilde{\boldsymbol{x}}\| \leq c_{2}, \quad\left\|\boldsymbol{v}_{n}\right\| \leq c_{3} .
$$

Using (34), (35) and (51), the bounding limits for the end effector and nullspace accelerations $\dot{\boldsymbol{\nu}}_{e}$ and $\dot{\boldsymbol{v}}_{n}$ are:

$$
\begin{aligned}
\left\|\dot{\boldsymbol{\nu}}_{e}\right\| & =\left\|-\boldsymbol{M}_{e}^{-1} \boldsymbol{C}_{e} \boldsymbol{\nu}_{e}-\boldsymbol{M}_{e}^{-1} \boldsymbol{D} \boldsymbol{\nu}_{e}-\boldsymbol{M}_{e}^{-1} \boldsymbol{J}_{\tilde{x} \nu}^{T} \boldsymbol{K} \tilde{\boldsymbol{x}}\right\| \\
& \leq \frac{\gamma_{C e}\left(c_{1}+c_{3}+h\right)+\lambda_{D, \max }}{\lambda_{M e, \min }} c_{1}+\frac{\lambda_{K, \text { max }} \mu_{1}}{\lambda_{M e, \min }} c_{2}=c_{4},
\end{aligned}
$$$$
\begin{aligned}
\left\|\dot{\boldsymbol{v}}_{n}\right\| & =\left\|-\boldsymbol{M}_{n}^{-1} \boldsymbol{C}_{n} \boldsymbol{v}_{n}-\boldsymbol{M}_{n}^{-1} \boldsymbol{D}_{n} \boldsymbol{v}_{n}\right\| \\
& \leq \frac{\gamma_{C n}\left(c_{1}+c_{3}+h\right)+\lambda_{D n, \max }}{\lambda_{M n, \min }} c_{3}=c_{5},
\end{aligned}
$$

with $c_{4}, c_{5}>0$. The bounding limit for $\dot{M}_{\boldsymbol{e}}$ is obtained from (33) as

$$
\left\|\dot{\boldsymbol{M}}_{e}\right\|=\left\|\boldsymbol{C}_{e}+\boldsymbol{C}_{e}^{T}\right\| \leq 2 \gamma_{C e}\|\chi\| \leq 2 \gamma_{C e}\left(c_{1}+c_{3}+h\right) .
$$

The bounding limit for $\dot{C}_{e}$ is obtained considering (51), (52):

$$
\begin{array}{r}
\left\|\dot{\boldsymbol{C}}_{e}\right\| \leq \gamma_{C e d 1}\|\boldsymbol{\chi}\|+\gamma_{C e d 2}\|\dot{\boldsymbol{\chi}}\| \\
\quad \leq \gamma_{C e d 1}\left(c_{1}+c_{3}+h\right)+\gamma_{C e d 2}\left(c_{4}+c_{5}\right) .
\end{array}
$$

The Barbalat's Lemma is reported here from [24] in form of corollary.

Barbalat's Lemma. If a differentiable function $f(t)$ has a finite limit as $t \rightarrow \infty$, and is such that $\ddot{f}$ exists and is bounded, then $\dot{f}(t) \rightarrow 0$ as $t \rightarrow 0$.

\section{REFERENCES}

[1] S. Dubowsky and E. Papadopoulos, "The kinematics, dynamics, and control of free-flying and free-floating space robotic systems," Robotics and Automation, IEEE Transactions on, vol. 9, no. 5, pp. 531-543, Oct 1993.

[2] E. Papadopoulos and S. Dubowsky, "Dynamic singularities in freefloating space manipulators," ASME J. Dyn.Syst., Meas., Contr.,115:1, vol. 115 , pp. $44-52,1993$.

[3] R. Lampariello and G. Hirzinger, "Generating feasible trajectories for autonomous on-orbit grasping of spinning debris in a useful time," in Intelligent Robots and Systems (IROS), 2013 IEEE/RSJ International Conference on, Nov 2013, pp. 5652-5659.
[4] Y. Umetani and K. Yoshida, "Continuous path control of space manipulators mounted on omv," Acta Astronautica, vol. 15, no. 12, pp. $981-986,1987$.

[5] $\_$, "Resolved motion rate control of space manipulators with generalized jacobian matrix," Robotics and Automation, IEEE Transactions on, vol. 5, no. 3, pp. 303-314, Jun 1989.

[6] Y. Masutani, F. Miyazaki, and S. Arimoto, "Sensory feedback control for space manipulators," in Robotics and Automation, IEEE International Conference on, May 1989, pp. 1346-1351 vol.3.

[7] D. Nenchev, Y. Umetani, and K. Yoshida, "Analysis of a redundant free-flying spacecraft/manipulator system," Robotics and Automation, IEEE Transactions on, vol. 8, no. 1, pp. 1-6, Feb 1992.

[8] K. Nanos and E. Papadopoulos, "On the use of free-floating space robots in the presence of angular momentum," Intelligent Service Robotics, vol. 4, no. 1, pp. 3-15, 2011.

[9] —_, "On cartesian motions with singularities avoidance for freefloating space robots," in Robotics and Automation (ICRA), 2012 IEEE International Conference on, May 2012, pp. 5398-5403.

[10] S. Xu, H. Wang, D. Zhang, and B. Yang, "Adaptive Reactionless Motion Control for Free-Floating Space Manipulators," ArXiv e-prints, Feb. 2014.

[11] H. Nakanishi and K. Yoshida, "Impedance Control for Free-flying Space Robots -Basic Equations and Applications-," in Intelligent Robots and Systems, 2006 IEEE/RSJ International Conference on, Oct 2006, pp. 3137-3142.

[12] A. Albu-Schäffer, C. Ott, and G. Hirzinger, "A unified passivitybased control framework for position, torque and impedance control of flexible joint robots," The International Journal of Robotics Research, vol. 26, no. 1, pp. 23-39, 2007.

[13] G. Garofalo, C. Ott, and A. Albu-Schaffer, "On the closed form computation of the dynamic matrices and their differentiations," in Intelligent Robots and Systems (IROS), 2013 IEEE/RSJ International Conference on, Nov 2013, pp. 2364-2359.

[14] C. Ott, Cartesian impedance control of redundant and flexible-joint robots. Springer, 2008.

[15] S. Stramigioli, V. Duindam, G. van Oort, and A. Goswami, "Compact Analysis of 3D Bipedal Gait Using Geometric Dynamics of Simplified Models," in Robotics and Automation, 2009. ICRA '09. IEEE International Conference on, May 2009, pp. 1978-1984.

[16] D. N. Nenchev, "Reaction null space of a multibody system with applications in robotics," Mechanical Sciences, vol. 4, no. 1, pp. 97$112,2013$.

[17] R. M. Murray, Z. Li, and S. S. Sastry, A Mathematical Introduction to Robotic Manipulation, 1st ed. Boca Raton, FL, USA: CRC Press, Inc., 1994.

[18] O. Khatib, "A unified approach for motion and force control of robot manipulators: The operational space formulation," Robotics and Automation, IEEE Journal of, vol. 3, no. 1, pp. 43-53, February 1987.

[19] G. Garofalo, B. Henze, J. Englsberger, and C. Ott, "On the inertially decoupled structure of the floating base robot dynamics," IFACPapersOnLine, vol. 48, no. 1, pp. 322 - 327, 2015, 8th Vienna International Conferenceon Mathematical ModellingMATHMOD 2015.

[20] C. Chen and I. Walker, "A consistent null-space based approach to inverse kinematics of redundant robots," in Robotics and Automation, IEEE International Conference on, May 1993, pp. 374-381 vol.3.

[21] J. Park, W. Chung, and Y. Youm, "On dynamical decoupling of kinematically redundant manipulators," in Intelligent Robots and Systems, 1999. IROS '99. Proceedings. 1999 IEEE/RSJ International Conference on, vol. 3, 1999, pp. 1495-1500 vol.3.

[22] K. Yoshida, N. Sashida, R. Kurazume, and Y. Umetani, "Modeling of collision dynamics for space free-floating links with extended generalized inertia tensor," in Robotics and Automation, IEEE International Conference on, May 1992, pp. 899-904 vol.1.

[23] J. Mulero-Martinez, "Uniform bounds of the coriolis/centripetal matrix of serial robot manipulators," Robotics, IEEE Transactions on, vol. 23, no. 5, pp. 1083-1089, Oct 2007.

[24] J.-J. E. Slotine and W. Li, Applied nonlinear control. Englewood Cliffs (N.J.): Prentice Hall, 1991. 